\title{
Throughput Maximization in Multicarrier Wireless Powered Relaying Networks
}

\author{
Panagiotis D. Diamantoulakis, Student Member, IEEE, Georgia D. Ntouni, Student Member, IEEE, \\ Koralia N. Pappi, Member, IEEE, George K. Karagiannidis, Fellow, IEEE, and Bayan S. Sharif, Senior Member, IEEE
}

\begin{abstract}
Dynamic power allocation and power splitting, in a multicarrier two-hop link with a wireless powered relay, is investigated. We first formulate the corresponding optimization problem, which consists of the joint optimization -in terms of achievable rate- of, 1) the dynamic power allocation among multiple channels and, 2) the selection of the power splitting ratio between information processing and energy harvesting at the relay, when amplify-and-forward is applied. This is a non-convex optimization problem, which is mapped to a convex one and optimally solved using one-dimensional search and dual decomposition, while a suboptimal efficient iterative method is also proposed. Simulations reveal a significant increase in the throughput, when comparing the proposed approach with two alternative power allocation schemes, while they verify the effectiveness of the fastconverging iterative solution.
\end{abstract}

Index Terms-Wireless power transfer, relay, power allocation, amplify-and-forward.

\section{INTRODUCTION}

W IRELESS power transfer is an upcoming energy harvesting technique, aiming to overcome the constraint of fixed energy supplies and provide self-sustainability to network nodes [1], [2]. Interestingly, wireless signals can be used for the simultaneous wireless information and power transfer (SWIPT). However, in practice, the node cannot harvest power and process the information from the received signal at the same time. Power splitting provides an efficient solution to this problem, as the received signal can be divided into two streams, one for information processing and one for energy harvesting [3]. SWIPT has also been investigated in the context of an orthogonal frequency division multiplexing (OFDM) point-topoint communication system [4], [5]. The employment of relays is known to enhance the quality of service $(\mathrm{QoS})$ and increase the network coverage, especially when there is no line of sight (LoS) between the source and the destination. However, when the relay nodes are placed in remote positions, it is difficult to supply them with power. To this end, the employment of energy harvesting renders the relaying nodes self-powered and independent [6]-[8], enabling communication between remote nodes when a direct transmission is not feasible.

Manuscript received February 4, 2015; revised March 23, 2015; accepted April 6, 2015. Date of publication April 17, 2015; date of current version August 20, 2015. This work was supported by the NPRP grant \# NPRP 6-13262-532 from the Qatar National Research Fund (a member of Qatar Foundation). The statements made herein are solely the responsibility of the authors. The associate editor coordinating the review of this paper and approving it for publication was I.-M. Kim.

P. D. Diamantoulakis, G. D. Ntouni, and K. N. Pappi are with the Aristotle University of Thessaloniki, Thessaloniki 54 124, Greece (e-mail: padiaman@ auth.gr; gntouni@auth.gr; kpappi@auth.gr).

G. K. Karagiannidis is with the Khalifa University, Abu Dhabi 127788, UAE, and with the Aristotle University of Thessaloniki, Thessaloniki 54 124, Greece (e-mail: geokarag@ieee.org).

B. S. Sharif is with the Khalifa University, Abu Dhabi 127788, UAE (e-mail: bayan.sharif@kustar.ac.ae).

Digital Object Identifier 10.1109/LWC.2015.2424237
In this work, a source and a destination are considered, which communicate through a wireless-powered relay over multiple channels. The amplify-and-forward (AF) protocol is considered, for low-complexity relay nodes, since it does not require decoding of the received signal. Note that AF relaying is included in the Long Term Evolution-Advanced (LTE-A) standard [9]. We further allow the relay to harvest energy from other sources -if available- such as solar power, wind etc., presenting a general optimization framework, which can accommodate various energy harvesting techniques, combined with wireless power transfer. Finally, for the problem of dynamic power splitting and power allocation on each of the available channels at the source and the relay, we take into account the maximization of the total throughput and the limitations of wireless power transfer process. To solve this problem, we propose two solutions: An optimal one and a fast-converging low-complexity iterative one.

\section{System Model}

A source (S), which communicates with a destination (D) via a relay (R) over $N$ independent channels, is assumed, where no LoS exists between $\mathrm{S}$ and $\mathrm{D}$. The relay is wireless powered and amplifies and forwards the received signals. The S-R and R-D channel gains over the $i^{\text {th }}$ channel are denoted by $h_{s, i}$ and $h_{r, i}$ respectively, and are considered independent complex Gaussian random variables with zero mean, i.e., $h_{s, i} \sim \mathcal{C N}\left(0, \sigma_{s, i}^{2}\right)$ and $h_{r, i} \sim \mathcal{C N}\left(0, \sigma_{r, i}^{2}\right)$, where $\sigma_{s, i}^{2}$ and $\sigma_{r, i}^{2}$ represent both path loss and shadowing. Furthermore, we assume that the relay performs energy harvesting by power splitting. The signal is split into two streams, and the power fraction, $\theta$, is used for harvesting, while the fraction $1-\theta$ is devoted to information processing. The received signal over the $i^{\text {th }}$ channel is [7]

$$
y_{r, i}=\sqrt{(1-\theta) P_{s, i}} h_{s, i} x_{s, i}+n_{r, i}
$$

where $P_{s, i}$ is the transmitted power by the source on the $i^{t h}$ channel, $x_{s, i}$ is the transmitted signal on the $i^{\text {th }}$ channel and $n_{r, i}$ is the additive white Gaussian noise (AWGN) at R, i.e., $n_{r, i} \sim \mathcal{C N}\left(0, W_{i} N_{0}\right)$, with $W_{i}$ being the $i^{t h}$ channel bandwidth. The total harvested power at $\mathrm{R}$ is

$$
P_{r t}=\eta \theta \sum_{i=1}^{N} P_{s, i}\left|h_{s, i}\right|^{2}+P_{0}
$$

where $0<\eta<1$ is the efficiency of the energy harvester and $P_{0}$ denotes the available power from other sources (apart from SWIPT) and/or other fixed power needs of R (except for retransmission). A case of special interest is when $P_{0}=0$, in which the relay utilizes all the harvested energy by SWIPT for retransmission.

The relay multiplies the received signal in (1) by

$$
G_{i}=\sqrt{\frac{P_{r, i}}{(1-\theta) P_{s, i}\left|h_{s, i}\right|^{2}+W_{i} N_{0}}}
$$


where, $P_{r, i}$, is the power transmitted by the relay over the $i^{t h}$ channel. Since R harvests energy from the first hop, which is then redistributed over the available channels, the transmission power values $P_{r, i}$ of $\mathrm{R}$ depend on the values $P_{s, i}$ of $\mathrm{S}$, the ratio $\theta$, the efficiency $\eta$ and the channel coefficients $h_{s, i}$. More specifically, it holds that

$$
\sum_{i=1}^{N} P_{r, i} \leq P_{r t}
$$

where $P_{r t}$ is given in (2). Thus, each $P_{r, i}$ needs to be optimally determined.

The signal which is received at the destination over the $i^{t h}$ channel is given by

$$
y_{d, i}=h_{r, i} G_{i} y_{r, i}+n_{d, i}
$$

where $n_{d, i}$ is the AWGN added at the destination with $n_{d, i} \sim$ $\mathcal{C N}\left(0, W_{i} N_{0}\right)$. If the signal-to-noise ratio (SNR) of the $\mathrm{S}-\mathrm{R}$ and $\mathrm{R}-\mathrm{D}$ communication links on the $i^{\text {th }}$ channel are

$$
\gamma_{s, i}=\frac{(1-\theta) P_{s, i}\left|h_{s, i}\right|^{2}}{W_{i} N_{0}}, \gamma_{r, i}=\frac{P_{r, i}\left|h_{r, i}\right|^{2}}{W_{i} N_{0}}
$$

respectively, then the total achievable rate is

$$
\mathcal{R}=\sum_{i=1}^{N} \frac{1}{2} W_{i} \log _{2}\left(1+\gamma_{i}\right),
$$

where the factor $\frac{1}{2}$ is due to the half duplex operation of the relay and $\gamma_{i}$ is the end-to-end SNR, given by

$$
\gamma_{i}=\frac{\gamma_{s, i} \gamma_{r, i}}{\gamma_{s, i}+\gamma_{r, i}+1}
$$

\section{Power Allocation \& Splitting Optimization}

In this section, we solve the joint dynamic power allocation and splitting optimization problem. The optimization is performed by a node with full channel state information (CSI). During estimation, $\mathrm{R}$ acquires CSI regarding the S-R link, while $\mathrm{D}$ acquires CSI regarding the R-D link, from pilot symbols sent by $\mathrm{S}$ and $\mathrm{R}$ respectively. The corresponding CSI is sent via feedback to the node which performs the optimization. The optimization problem can be defined as

$$
\begin{array}{ll}
\underset{\mathcal{P}_{s}, \theta, \mathcal{P}_{r}}{\max } & \mathcal{R} \\
\text { s.t. } & C_{1}: \sum_{i=1}^{N} P_{r, i} \leq P_{r t}, C_{2}: \sum_{i=1}^{N} P_{r, i} \leq P_{r m} \\
& C_{3}: \sum_{i=1}^{N} P_{s, i} \leq P_{s m}, C_{4}: 0 \leq \theta \leq 1 \\
& C_{5}: P_{s, i} \geq 0, \forall i, C_{6}: P_{r, i} \geq 0, \forall i
\end{array}
$$

where $\mathcal{P}_{s}$ and $\mathcal{P}_{r}$ are the sets of the allocated power, $P_{s, i}$ and $P_{r, i}$, respectively. Constraint $C_{1}$ represents the limited harvested power which is available for retransmission. Constraints $C_{2}$ and $C_{3}$ include the hardware and regulations limitations $P_{r m}$ and $P_{s m}$ on the total transmitted power by $\mathrm{R}$ and $\mathrm{S}$, respectively.

\section{A. Optimization With One-dimensional Search for $\theta$}

The optimization problem (9) is non-convex, therefore the complexity to solve it is high, mainly due to the existence of the power splitting ratio $\theta$, which couples the power allocation variables and results in a non-convex function. To overcome this limitation and derive a tractable power allocation algorithm, we perform a full search with respect to $\theta$, as in [4]. In practice, to solve (9), we discretize the range of $\theta \in[0,1]$ in $K+1$ equally spaced intervals, i.e. $\theta \in\left\{0, \theta_{1}, \theta_{2}, \ldots, \theta_{K, 1}\right\}$. For the interval width, $T=\theta_{n}-\theta_{n-1}$, it holds that $0<T<<1$, while for each $\theta_{n}, n=1, \ldots, K,(9)$ has to be solved (values 0 and 1 are excluded from the search because they lead to $R_{\text {tot }}=0$ ). Therefore, the complexity of one-dimensional search is proportional to the number of value intervals for $\theta$, while it is optimal only for infinitely small value intervals. However, even with the aid of the above, the optimization problem in (9) is still nonconvex, so we proceed to the following well-known approximation for the end-to-end SNR, which has been shown to be tight, especially in the medium and high SNR region [10]. Thus,

$$
\begin{aligned}
& \gamma_{i}=\frac{\gamma_{s, i} \gamma_{r, i}}{\gamma_{s, i}+\gamma_{r, i}+1} \simeq \frac{\gamma_{s, i} \gamma_{r, i}}{\gamma_{s, i}+\gamma_{r, i}}=\tilde{\gamma}_{i} \\
& \mathcal{R} \simeq \tilde{\mathcal{R}}=\sum_{i=1}^{N} \frac{1}{2} W_{i} \log _{2}\left(1+\tilde{\gamma}_{i}\right) .
\end{aligned}
$$

Now, for a specific value of $\theta$, problem (9) simplifies to

$$
\begin{array}{ll}
\underset{\mathcal{P}_{s}, \mathcal{P}_{r}}{\max } & \tilde{\mathcal{R}} \\
\text { s.t. } & C_{1}, C_{2}, C_{3}, C_{5}, C_{6}
\end{array}
$$

which is jointly concave with respect to the optimization variables, since the Hessian matrix of its objective function is negative semi-definite. Moreover, it satisfies Slater's constraint qualification, and, thus, it can now be optimally and efficiently solved with dual decomposition, since the duality gap between the dual and the primal solution is zero [11]. More importantly, it is guaranteed that its global optimum solution can now be obtained in polynomial time.

The Lagrangian of the primal problem (12) is given by

$$
\begin{aligned}
\mathcal{L}=\tilde{\mathcal{R}}-l_{1}\left(\sum_{i=1}^{N} P_{r, i}-\right. & \left.P_{r t}\right)-l_{2}\left(\sum_{i=1}^{N} P_{r, i}-P_{r m}\right) \\
& -l_{3}\left(\sum_{i=1}^{N} P_{s, i}-P_{s m}\right)
\end{aligned}
$$

where $l_{1}, l_{2}, l_{3} \geq 0$ are the Lagrange multipliers (LMs) of the constraints $C_{1}, C_{2}, C_{3}$, correspondingly. The constraints $C_{5}$ and $C_{6}$ will be absorbed into the Karush-Kuhn-Tucker (KKT) conditions and, thus, the dual problem is given by

$$
\min _{l_{1}, l_{2}, l_{3}} \max _{\mathcal{P}_{s}, \mathcal{P}_{r}} \mathcal{L}
$$

The dual problem in (14) can be recursively solved in two consecutive layers, namely Layer 1 and Layer 2. In each recursion, the subproblem of power allocation at $S$ and $R$ is solved in Layer 1 by using the KKT conditions for a fixed set of LMs, which are then updated in Layer 2. For this purpose, the gradient method is used, which enables the parallelized solution of $N$ identically structured problems, corresponding to the optimization of $P_{s, i}$ and $P_{r, i}$ (Layer 1) and requiring only knowledge of the updated values of the LMs. This two-layer approach, which converges after a reasonable number of recursions, reduces considerably the required computational and memory resources. The two layers are explained in detail below. 
Layer 1: Using the KKT conditions, the optimal power allocation on the $i^{t h}$ channel is given by

$$
\begin{aligned}
& P_{s, i}=\left[P_{s, i} \in \mathbb{R}: \frac{\partial \mathcal{L}}{\partial P_{s, i}}=0 \cap \frac{\partial \mathcal{L}}{\partial P_{r, i}}=0\right]^{+}, \\
& P_{r, i}=\left[P_{r, i} \in \mathbb{R}: \frac{\partial \mathcal{L}}{\partial P_{s, i}}=0 \cap \frac{\partial \mathcal{L}}{\partial P_{r, i}}=0\right]^{+},
\end{aligned}
$$

where $[\cdot]^{+}=\max (\cdot, 0)$. The analytical expressions of the optimal power allocation can be derived with simple mathematical calculations, which are omitted due to space limitations. It is remarkable that the allocated power in each channel can be calculated in parallel, which further reduces the complexity of the proposed solution.

Layer 2: Since the dual function is differentiable, the gradient method can be used to update the LMs as follows

$$
\begin{aligned}
& l_{1}(t+1)=\left[l_{1}(t)-\alpha_{1}(t)\left(P_{r t}-\sum_{i=1}^{N} P_{r, i}\right)\right]^{+}, \\
& l_{2}(t+1)=\left[l_{2}(t)-\alpha_{2}(t)\left(P_{r m}-\sum_{i=1}^{N} P_{r, i}\right)\right]^{+}, \\
& l_{3}(t+1)=\left[l_{3}(t)-\alpha_{3}(t)\left(P_{s m}-\sum_{i=1}^{N} P_{s, i}\right)\right]^{+},
\end{aligned}
$$

where index $t>0$ is the recursion index and $\alpha_{j}(t), j \in$ $\{1,2,3\}$ are positive step sizes, chosen to satisfy the diminishing step size rules [12]. Since the transformed problem is concave, it is guaranteed that the iteration between the two layers converges to the primal optimal solution [11], [12].

\section{B. Iterative Solution}

In this subsection, inspired by the alternating optimization [13], we introduce a suboptimal iterative solution to solve (9). In each iteration, we first solve problem (12) with respect to $\mathcal{P}_{s}$ and $\mathcal{P}_{r}$, for a specific $\theta$ (a given initialization value). Then, the derived solution for $\mathcal{P}_{s}$ is used as an input to solve the following problem

$$
\begin{array}{ll}
\max _{\theta, \mathcal{P}_{r}} & \tilde{R} \\
\text { s.t. } & C_{1}, C_{2}, C_{4}, C_{6} .
\end{array}
$$

Note that, $\mathcal{P}_{r}$ is also optimized in (17) and is not considered as given, using the solution of (12) is a dependent variable on $\mathcal{P}_{s}$ and $\theta$ and, thus, it is jointly optimized with each of these two variables, in each step. The new value of $\theta$, which is given by the solution of (17), is used in the next iteration to find the new values for $\mathcal{P}_{s}$ and $\mathcal{P}_{r}$. Each iteration is a sequential solution of problems (12) and (17). Thus, the complexity of the iterative method is proportional to the number of iterations.

The optimization problem in (17) can be solved similarly to that in (12), since it is also concave with respect to the optimization variables. Specifically, the Lagrangian of the primal solution is given by

$$
\Lambda=\tilde{\mathcal{R}}-\lambda_{1}\left(\sum_{i=1}^{N} P_{r, i}-P_{r t}\right)-\lambda_{2}\left(\sum_{i=1}^{N} P_{r, i}-P_{r m}\right)
$$

where $\lambda_{1}, \lambda_{2} \geq 0$ are the LMs.

The dual problem in this case is defined as

$$
\min _{\lambda_{1}, \lambda_{2}} \max _{\theta, \mathcal{P}_{r}} \Lambda \text {. }
$$

Thus, an approach of two layers can be also used to find the optimum solution.

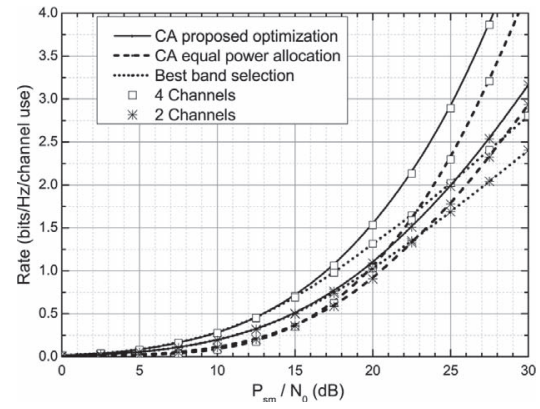

Fig. 1. Rate against SNR for different number of channels.

Layer 1: Using again the KKT conditions, the optimal values of $\theta$ and $\mathcal{P}_{r}$ are given by

$$
\begin{aligned}
\theta & =\left[\theta \in \mathbb{R}: \frac{\partial \Lambda}{\partial \theta}=0 \cap \frac{\partial \Lambda}{\partial P_{r, i}}=0, \forall i\right]_{0}^{1}, \\
P_{r, i} & =\left[P_{r, i} \in \mathbb{R}: \frac{\partial \Lambda}{\partial \theta}=0 \cap \frac{\partial \Lambda}{\partial P_{r, i}}=0, \forall i\right]^{+},
\end{aligned}
$$

where $[\cdot]_{0}^{1}=\max (\min (\cdot, 1), 0)$.

Layer 2: The LMs are updated by

$$
\begin{aligned}
& \lambda_{1}(t+1)=\left[\lambda_{1}(t)-\beta_{1}(t)\left(P_{r t}-\sum_{i=1}^{N} P_{r, i}\right)\right]^{+}, \\
& \lambda_{2}(t+1)=\left[\lambda_{2}(t)-\beta_{2}(t)\left(P_{r m}-\sum_{i=1}^{N} P_{r, i}\right)\right]^{+},
\end{aligned}
$$

where $\beta_{j}(t), j \in\{1,2\}$ are again positive step sizes. Note that, since $P_{r t}$ is dependent on $\theta$ as shown in (2), it is updated in each recursion.

\section{Simulations AND Discussion}

In this section, we present results from simulations for a system which utilizes two or four channels of unitary bandwidth, i.e., $W_{i}=1 \mathrm{~Hz}, \forall i$. Furthermore, we focus on the case where the relay harvests energy only via SWIPT and utilizes that for retransmission, i.e., $P_{0}=0$. We also assume that $P_{r m}=P_{s m}$. The coordinates of the source and destination are $(-1,0)$ and $(1,0)$, respectively, while the relay is placed at $(-0.25,0.5)$. We assume a bounded path loss model, for which $\sigma_{s, i}^{2}=\frac{1}{1+d_{s r}^{a_{i}}}$ and $\sigma_{r, i}^{2}=\frac{1}{1+d_{r d}^{a_{i}}}$, where $a_{i}$ is the path loss exponent of the $i^{\text {th }}$ channel, as in [8]. This model ensures that the path loss model is valid even for distance values lower than $1 \mathrm{~m} \mathrm{[14].}$ Specifically, we consider that $a_{1}=2$ and $a_{2}=2.5$, to capture the different propagation characteristics of each channel. When four channels are used, we further assume that $a_{3}=3$ and $a_{4}=$ 3.5. Finally, the efficiency of the energy harvester is set to $\eta=$ 0.3 , as a worst case, capturing the effects of low-cost hardware.

In Fig. 1, the average achievable rate of the proposed optimization is illustrated, for different values of the power ratio, $\frac{P_{s m}}{N_{0}}$. In conjunction with the proposed method, two other power allocation strategies are illustrated for comparison: a) equal power splitting between the channels, i.e., $P_{s, i}=\frac{P_{s m}}{N} \forall i$ and $P_{r, i}=\frac{\min \left(P_{r t}, P_{r m}\right)}{N} \forall i$, and b) selection of only the best channel $j$ (the one which maximizes the achievable rate if only one is selected) for each realization, i.e., $P_{s, j}=P_{s m}, P_{r, j}=$ $\min \left(P_{r t}, P_{r m}\right)$, and $P_{s, i}=P_{r, i}=0 \forall i \neq j$. For all methods, the optimal power splitting ratio is dynamically computed. The proposed power allocation clearly outperforms both schemes, 


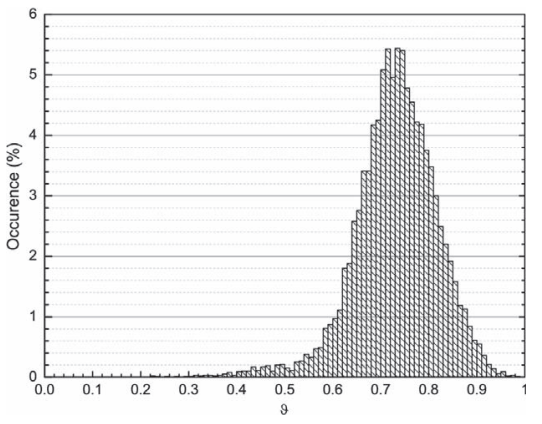

Fig. 2. Occurrence of values for the optimal $\theta$ in 100 intervals between $\theta=0$ and $\theta=1$.

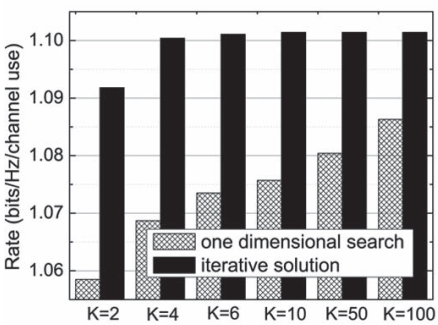

(a)

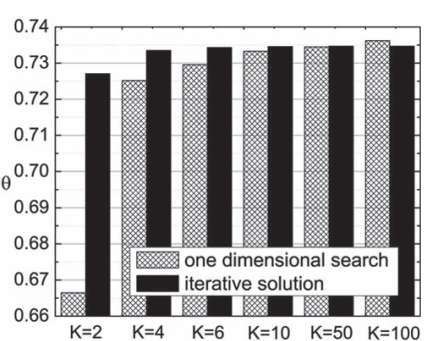

(b)
Fig. 3. Comparison of the two optimization methods for $K$ optimization. (a) Rate. (b) $\theta$.

especially when the number of channels increases. For low values of the power ratio, the results for the proposed method and the best channel selection are very close, which implies that the dynamic power allocation preferably allocates more power over the best channel. However, for power ratio values over $15 \mathrm{~dB}$, the proposed method clearly outperforms the best channel selection, while it displays a performance gain gap over the equal power allocation method of about $1 \mathrm{~dB}$ or $2 \mathrm{~dB}$ when 2 or 4 channels are used, respectively.

In Fig. 2, the distribution of the optimal value of $\theta$ is illustrated when 2 channels are used, for 10,000 channel realizations. The transmitted power to noise ratio is set to $\frac{P_{s m}}{N_{0}}=$ $20 \mathrm{~dB}$ and the values of $\theta$ are grouped in intervals of width equal to 0.01 . One can observe that the values of optimal $\theta$ which occur more often are within 0.71 and 0.75 , while more than $90 \%$ of the channel realizations lead to an optimal value for $\theta$ in the interval between 0.6 and 0.9 . These values, which correspond to harvesting more than $60 \%$ of the received power, are expected due to the relative position of the network nodes, since more power is needed for transmission over the second hop which is of longer distance.

In Fig. 3, the two proposed optimization methods, i.e., the one-dimensional search for $\theta$ and the iterative solution, are directly compared in terms of complexity and convergence, for $\frac{P_{s m}}{N_{0}}=20 \mathrm{~dB}$, for two utilized channels and for the same number of optimization operations. As it can be observed, the iterative method is suboptimal, but it converges fast, while it outperforms the one-dimensional search for low number of operations. More specifically, the solution of the one-dimensional search reaches closer to the optimum when more values of $\theta$ are searched, i.e., for high values of $K$. Each value of $\theta$ which is searched corresponds to solving one optimization problem in (12). Similarly, the iterative method converges to the optimal solution as more iterations are performed. Each iteration comprises two steps, i.e., one solution of (12) and one solution of (17). Thus, for the sake of a fair complexity comparison, $K$ values of $\theta$ in the one-dimensional search are compared to $\frac{K}{2}$ iterations of the iterative method, corresponding to solving $K$ optimizations. Specifically, in Fig. 3(a), the rate which is achieved after $K=2,4,6,10,50,100$ optimization runs is compared. It is easily observed that the iterative solution converges faster than the one-dimensional search, since in the latter, $\theta$ is restricted to specific quantized values. The resulting rate for the iterative method reaches its optimal value for 5 iterations $(K=10)$ and remains unchanged afterwards. Note, however, that both methods achieve solutions for the rate that are less than $4 \%$ lower than the optimal value, even for $K=2$. Similar observations can be made in Fig. 3(b), where the optimal values of $\theta$ are compared.

\section{Conclusion}

In this paper, the total achievable rate of a two-hop multicarrier link was maximized, with respect to the power allocation at the source and the relay, and the power splitting ratio for energy harvesting at the relay. Two different solution methods were proposed, while they were compared in terms of complexity and convergence. It was shown that the proposed strategy with any of the two methods leads to a notable increase of the total achievable rate, compared to equal power allocation or best channel selection. The ideas developed in this paper can be extended in several directions. The presented results can also be considered as a benchmark for all other practical cases, such as imperfect CSI or imperfect feedback.

\section{REFERENCES}

[1] L. Varshney, "Transporting Information and Energy Simultaneously," in Proc. IEEE Int. Symp. Inf. Theory, Jul. 2008, pp. 1612-1616.

[2] P. Grover and A. Sahai, "Shannon meets tesla: Wireless information and power transfer," in Proc. IEEE Int. Symp. Inf. Theory, 2010, pp. 2363-2367.

[3] L. Liu, R. Zhang, and K. C. Chua, "Wireless information and power transfer: A dynamic power splitting approach," IEEE Trans. Commun., vol. 61, no. 9, pp. 3990-4001, Sep. 2013.

[4] D. W. K. Ng, E. S. Lo, and R. Schober, "Energy-efficient power allocation in OFDM Systems with wireless information and power transfer," in Proc. IEEE ICC, Jun. 2013, pp. 4125-4130.

[5] D. W. K. Ng and R. Schober, "Spectral efficient optimization in OFDM systems with wireless information and power transfer," in Proc. 21st EUSIPCO, Sep. 2013, pp. 1-5.

[6] D. S. Michalopoulos, H. A. Suraweera, and R. Schober, "Relay selection for simultaneous information transmission and wireless energy transfer: A tradeoff perspective," IEEE J. Sel. Areas Commun., to be published.

[7] Z. Ding, S. M. Perlaza, I. Esnaola, and H. V. Poor, "Power allocation strategies in energy harvesting wireless cooperative networks," IEEE Trans. Wireless Commun., vol. 13, no. 2, pp. 846-860, Feb. 2014.

[8] Z. Ding, I. Krikidis, B. Sharif, and H. V. Poor, "Wireless information and power transfer in cooperative networks with spatially random relays," IEEE Trans. Wireless Commun., vol. 13, no. 8, pp. 4440-4453, Aug. 2014.

[9] "Evolved Universal Terrestrial Radio Access (E-UTRA); Relay Architectures for E-UTRA (LTE-Advanced), "3rd Generation Partnership Project, Sophia-Antipolis Cedex, France, 3GPP TR 36.806.

[10] M. O. Hasna and M. S. Alouini, "Performance analysis of two-hop relayed transmission over Rayleigh fading channels," in Proc. IEEE VTC, Vancouver, BC, Canada, Sep. 2002, pp. 1992-1996.

[11] S. Boyd and L. Vandenberghe, Convex Optimization. Cambridge, U.K.: Cambridge Univ. Press, 2004.

[12] S. Boyd, L. Xiao, and A. Mutapcic, "Subgradient Methods," Stanford Univ. Stanford, CA, USA, Notes EE392o, 2003-2004.

[13] J. C. Bezdek and R. Hathaway, "Some notes on alternating optimization," in Proc. Int. Conf. AFSS, 2002, pp. 187-195.

[14] M. Haenggi, Stochastic Geometry for Wireless Networks. Cambridge, U.K.: Cambridge Univ. Press, 2012. 